\title{
Mesh Antennas with Reduced Size
}

\author{
Tze-Hsuan Chang*, Cheng-Wei Lan, and Jean-Fu Kiang \\ Department of Electrical Engineering and \\ Graduate Institute of Communication Engineering \\ National Taiwan University, Taipei, Taiwan, ROC \\ E-mail: jfkiang@cc.ee.ntu.edu.tw
}

\section{Abstract}

In this paper, we propose a new design based on rectangular microstrip antenna, which has lower resonant frequencies or smaller size. Although slightly larger cross-polarization may be induced, but it can be reduced by properly choosing the wire direction. The resonant frequency can be tuned by adjusting the mesh density.

\section{Introduction}

Major characteristics of microstrip antennas are their low efficiency, high $Q$-factor, poor polarization purity and narrow frequency bandwidth. In certain applications, narrow bandwidths are desirable. Lots of researches focus on the shape of patches or the aperture pattern carved on them to obtain broadband or dual-band operation, or circular polarization.

Apertures on the patch can be used to alter or constrain the current flow direction. If the direction of mesh wires follows the surface current distribution of a specific resonant mode, that resonant mode will be excited. If the mesh pattern or the wire perturbs the current distribution, the associated resonant frequency will be varied. In both cases, the radiation pattern and polarization will be affected. However, mesh patterns can be designed carefully to reduce cross-polarization or change the polarization.

Embedding wider arc-shaped slot near the edge of circular patch or loading a $\mathrm{U}$-shaped slot in the microstrip patches of various shapes can achieve broadband operation. If the slot is arranged to be parallel to the current direction of a specific resonant mode, its characteristics will be affected slightly. If the slot perturbs the current of another mode, its resonant frequency can be significantly reduced. Such two resonant frequencies can be brought close to each other and form a wide band [1]

Substrate with thicker and lower dielectric constant can render stronger fringing field and make the approximation of magnetic wall less accurate. The internal fields have less constraint over a wider bandwidth. However, thicker substrate implies longer feeding probe which induces larger inductance and hence narrower bandwidth $(<10 \%)$. Etching a small circular slot on the patch 
around the feed location can induce capacitance to compensate for the probe inductance and increase the bandwidth (16\%) [1].

Inserting a reactive loading on the microstrip antenna can also obtain wide bandwidth. For example, a capacitor can be used as a current sink. Placing a capacitor properly can increase the current path length of a specific resonant mode while not disturbing the other modes. Hence, the perturbed mode will have lower resonant frequency. If the resonant frequency is brought close to the lower one, a wide bandwidth can be achieved [2]

Fig.1 shows the current directions on a wire grid array at resonant frequency. The current on vertical wires are in phase, while each horizontal segment supports a full wave. The vertically polarized field components can be adjusted to add while the horizontally ones cancel. As more loops are added to the grid configuration, the magnitude of the cross-polarized wave is reduced, and the wave radiated by vertical segments is enhanced. The cross-polarization levels can be further reduced by the presence of antiphase couplets and additional null plane [3]. The characteristic line impedances of the vertical elements, which are approximately inversely proportional to width, determine the current of that element. By varying the width of selected grid elements, the current amplitude can be controlled and both co-polarization and crosspolarization levels become adjustable. Additional suppression would occur if element factor and edge effects were included.

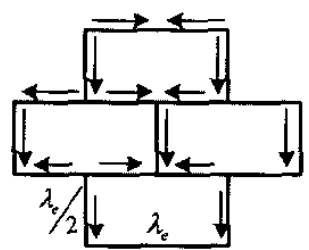

Figure 1: Current distribution of wire grid array antenna at resonant frequency. Currents on horizontal segment pair cancel each other at far field, and currents on vertical segments are in phase and their fields add constructively.

\section{Results and Conclusions}

According to the cavity model and empirical formulas [4], an operating frequency of $1.8 \mathrm{GHz}$ can be obtained by a rectangular patch with about $40 \mathrm{~mm}$ of length on a substrate with the relative dielectric constant of 4.2 and thickness of $1.6 \mathrm{~mm}$. The width of the patch is $30 \mathrm{~mm}$. A microstrip line of $50 \Omega$ (about 3 $\mathrm{mm}$ wide) is used to feed the patch antenna. To match the input impedance of the patch, a notch of $17 \mathrm{~mm}$ in depth is extended into the patch [4]. In order not to affect the characteristic impedance of the feed line, a side-gap, $S$, between feed line and patch is chosen to be the same as the width of feed line, W0, as shown in Fig.2(a). Fig.3 shows the HFSS simulation result of return loss of the 
patch antenna. The resonant frequency is near $1.8 \mathrm{GHz}$, the return loss is down to $-25 \mathrm{~dB}$, and the $10 \mathrm{~dB}$ bandwidth is very narrow as expected.

Next, array of rectangular apertures is opened on the patch to form a mesh antenna as shown in Fig.2 (b). All the parameters are the same as for the solid patch just described, including the feeding mechanism. The surface current of resonant mode on the mesh antenna has to flow along the metal strip, and the current path is slightly longer than that on the solid patch. Thus, the resonant frequency is only significantly reduced. Notice that the pattern of the metal strips is very similar to the current flowing paths of fundamental resonant mode.

Knowing the direction of current flow of the fundamental resonant mode, we arrange the metal strips to be aligned with the current direction of the fundamental mode, but in a crisscross pattern as shown in Fig.2(c). Strips in the perpendicular direction remain the same. As a result, the current flow of the original resonant mode needs to detour along longer paths, which perturbs the current distribution and thus lower the resonant frequency significantly, as shown in Fig.4. The resonant frequency of the solid patch, $1.83 \mathrm{GHz}$, is reduced to $1.59 \mathrm{GHz}$ for longitudinal meander mesh antenna. The extent of reduction depends on the density of longitudinal segments. However, the resonant current flowing in meander paths has orthogonal components and hence incurs crosspolarization.

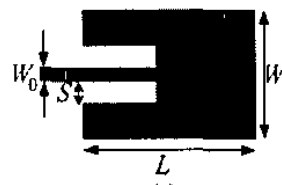

(a)

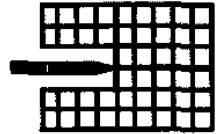

(b)

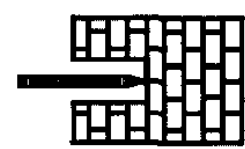

(c)

Figure 2 (a) Configuration of rectangular patch, $W=3 \mathrm{~mm}, L=4 \mathrm{~mm}, W_{0}=3 \mathrm{~mm}, X=17 \mathrm{~mm}$ $S=3 \mathrm{~mm}$, thickness of FR4 substrate is $1.6 \mathrm{~mm}$, (b) regular mesh antenna, (c) longitudinal meander mesh antenna. 


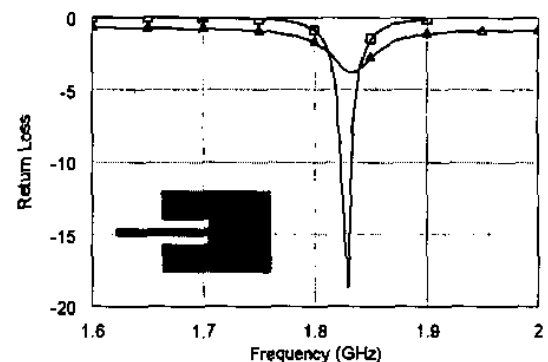

Figure 3 Retum loss of rectangular patch, the resonant frequency is about $1.835 \mathrm{GHz}$. $-\Delta^{--}$ HFSS simulation result, $\square$ - - measurement result.

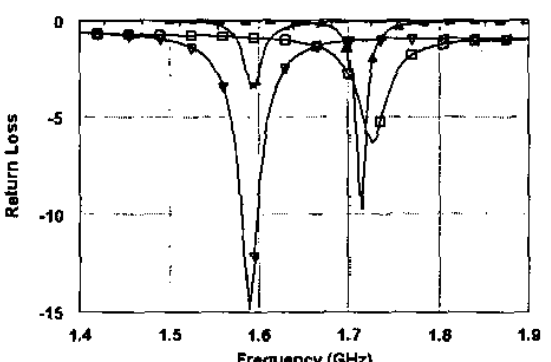

Figure 4 Return loss of regular mesh and longitudinal meandered mesh. - $\nabla-:$ longitudinal meandered mesh measurement result, -+: longitudinal meandered mesh HFSS simulation result, - - -: regular mesh HFSS simulation result, $-\triangle$-: regular mesh measurernent result.

Acknowledgment: This work is sponsored by the National Science Council, ROC, under contract NSC 92-2213-E-002-070.

Reference

[1] J. H. Lu, "Broadband dual-frequency operation of circular patch antennas and arrays with a pair of L-shaped slots," IEEE Trans. Antennas Propagat., vol.51, no.5, pp.1018-1023, May 2003.

[2] K. M. Luk, C. L. Mak, Y. L. Chow, and K. F. Lee, "Broadband microstrip patch antenna," Electron. Lett., vol.34, no.15, pp.1442-1443, July 23rd 1998.

[3] R. Conti, J. Toth, T. Dowling, and J. Weiss, "The wire grid microstrip antenna," IEEE Trans. Antennas Propagat., vol.29, no.1, pp.157-166, Jan. 1981.

[4] C. A. Balanis, Antenna Theory, Analysis and Design, John Wiley, 1997. 